\title{
Gastrokine 1 inhibits gastric cancer cell migration and invasion by downregulating RhoA expression
}

\author{
Jung Hwan Yoon ${ }^{1} \cdot$ Won Suk Choi ${ }^{1} \cdot$ Olga Kim $^{1} \cdot$ Byung Joon Choi $^{2} \cdot$ \\ Suk Woo Nam ${ }^{1} \cdot$ Jung Young Lee ${ }^{1} \cdot$ Won Sang Park ${ }^{1}$
}

Received: 19 February 2016/Accepted: 17 May 2016/Published online: 1 June 2016

(c) The International Gastric Cancer Association and The Japanese Gastric Cancer Association 2016

\begin{abstract}
Background We investigated whether GKN1, a gastric tumor suppressor, contributes to the progression of gastric cancer by regulating RhoA expression.

Methods We analyzed the expression of GKN1, RhoA, miR-185, and miR-34a in 35 gastric cancer tissues, and compared their expression with T category and TNM stage. Cell migration and invasion, as well as the expression of epithelial-to-mesenchymal transition (EMT)-related proteins, were assessed in GKNI- and RhoA small interfering RNA (siRhoA)-transfected and recombinant-GKN1-treated AGS and MKN1 gastric cancer cells.

Results Expression of RhoA protein and messenger RNA (mRNA) was increased in $15(42.9 \%)$ and $17(48.6 \%)$ of 35 gastric cancer tissues respectively, and was associated with higher T category and TNM stage. GKN1 expression was significantly decreased in 27 gastric cancers $(77.1 \%)$ with a higher $\mathrm{T}$ category, and was inversely correlated with RhoA mRNA expression. In AGS and MKN1 cells, GKN1 expression increased miR-185 and miR-34a expression and reduced RhoA mRNA and protein expression. A positive relationship between GKN1 and miR-34a and miR-185 expression and an inverse relationship between miR-34a
\end{abstract}

Jung Hwan Yoon and Won Suk Choi contributed equally to this study.

Won Sang Park

wonsang@catholic.ac.kr

1 Department of Pathology, College of Medicine, The Catholic University of Korea, 505 Banpo-dong, Seocho-gu, Seoul 137-701, Korea

2 Department of Pediatrics, College of Medicine, The Catholic University of Korea, 505 Banpo-dong, Seocho-gu, Seoul 137-701, Korea and RhoA expression were observed in gastric cancer tissues. Cell migration and invasiveness were markedly decreased in GKN1- and siRhoA-transfected cells. GKN1 expression and silencing of RhoA decreased the expression of the proteins Snail, Slug, and vimentin. Furthermore, miR-185 and miR-34a silencing in MKN1 cells transfected with GKN1 stimulated cell migration and invasion, and increased the expression of EMT-related proteins.

Conclusion Our data suggest that GKN1 may inhibit gastric cancer cell migration and invasion by downregulating RhoA expression in a miR-185- and miR-34a-dependent manner.

Keywords Gastrokine 1 - Gastric cancer - RhoA . Invasion $\cdot$ miR-185

\section{Introduction}

Gastric cancer is the leading cause of cancer death in the world, with an estimated 723,000 deaths in 2012, accounting for $8.8 \%$ of total deaths from cancer $[1,2]$. In spite of improving surgical resection in the early stages of the disease, the prognosis of patients with gastric cancer remains poor, due to resistance to traditional therapies and metastasis [3, 4]. Therefore, understanding the molecular mechanisms underlying gastric cancer metastasis may provide new insights into the development of novel therapies and improve patient prognosis.

A downregulation of E-cadherin, which promotes cellcell contact, and an upregulation of mesenchymal markers, such as vimentin, are characteristics of epithelial-to-mesenchymal transition (EMT). EMT molecular reprogramming causes cells to switch from an immobile epithelial phenotype to a motile mesenchymal phenotype, leading to 
increased cell migration and invasion [5]. Although various signaling pathways associated with EMT during tumor progression have been studied [6-8], the signaling mechanisms involved in EMT in gastric cancer cells remain unclear.

Gastrokine 1 (GKN1) is exclusively expressed in the gastric epithelium, and is downregulated in gastric cancer [9]. GKN1 is involved in the maturation and integrity of gastric mucosal epithelial cells [10]. Previously, we reported that GKN1 possesses miR-185-dependent and miR-185-independent mechanisms, for chromatic and DNA epigenetic modifications, thereby regulating the cell cycle [11]. In addition, GKN1 inhibits EMT by downregulating reactive oxygen species production and the phosphatidylinositol-3-kinase (PI3K)/Akt signaling pathway, as well as by inactivating nuclear factor $\mathrm{\kappa B}$ dependent matrix metalloproteinases $[12,13]$. RhoA is a key member of the Rho family of small GTP-binding proteins and is a master regulator of actin-myosin-dependent cell contractility and cellular motility $[14,15]$. Numerous studies have found that RhoA regulates the signaling pathways related to gene expression, cell proliferation, and cell migration [16-18].

In the present study, to better clarify a possible mechanism for the inhibitory effects of GKN1 on gastric cancer progression, we assessed whether GKN1 suppresses gastric cancer cell motility and invasion by downregulating RhoA expression in gastric cancer cell lines and tissues.

\section{Materials and methods}

\section{Mutational analysis}

Thirty-five frozen gastric cancers and corresponding mucosal tissues were obtained from the Chonnam National University Hwasun Hospital National Biobank of Korea, a member of the National Biobank of Korea, which is supported by the Ministry of Health, Welfare, and Family Affairs. For gastric cancers, 23 cases were of the intestinal type and 12 were of the diffuse type. The mean cancer size was $6.5 \mathrm{~cm}$. Freshly excised primary gastric cancer tissues were cut with a razor blade. The cut surfaces of each cancer sample were cryosectioned, and the 5-10- $\mu \mathrm{m}$ sections were screened by a pathologist after they had been stained with hematoxylin and eosin. Gastric cancer tissues containing more than $60 \%$ carcinoma cells were included in this study. Approval was obtained from the Institutional Review Board of the College of Medicine of the Catholic University of Korea (CUMC09U089). There was no evidence of familial cancer in any of the patients.

To investigate the mutation of the RhoA gene, genomic DNAs from each tumor cell and the corresponding normal mucosal cells were amplified with two sets of primers covering the entire coding region of the RhoA gene. The primer sequences are described in Table 1. Each polymerase chain reaction (PCR) procedure was performed under standard conditions, as previously described [19]. The reaction mixture was denatured at $94{ }^{\circ} \mathrm{C}$ for $12 \mathrm{~min}$ and then incubated for 35 cycles (denaturing at $94{ }^{\circ} \mathrm{C}$ for $40 \mathrm{~s}$, annealing at $50-54{ }^{\circ} \mathrm{C}$ for $40 \mathrm{~s}$, and extension at $72{ }^{\circ} \mathrm{C}$ for $40 \mathrm{~s}$ ). A final extension step at $72{ }^{\circ} \mathrm{C}$ was performed for $5 \mathrm{~min}$. Sequencing of PCR products was done with a cycle sequencing kit (PerkinElmer, Foster City, CA, USA) according to the manufacturer's protocol.

\section{Messenger RNA expression of GKN1 and RhoA}

For messenger RNA (mRNA) expression, complementary DNA (cDNA) was synthesized with a reverse transcription kit from Roche Molecular Systems (Mannheim, Germany) according to the manufacturer's protocol. For quantitative PCR (qRCR), 50 ng cDNA was amplified with Fullvelocity SYBR Green qPCR master mix (Stratagene, La Jolla, CA, USA) and each of the forward and reverse primers at $20 \mathrm{pmol} / \mu \mathrm{l}$ with use of a Stratagene Mx 3000P qPCR system with techniques previously described [19]. The specific oligonucleotide primers for mRNA were synthesized according to published information on the GKN1 gene, as described in Table 1. To ensure the fidelity of DNA and mRNA extraction and reverse transcription, all samples were subjected to PCR amplification with oligonucleotide primers specific for the constitutively expressed glyceraldehyde 3-phosphate dehydrogenase gene $(G A P D H)$ and normalized. The primer sequences of GAPDH are described in Table 1. Primers for SYBR Green analysis were designed on the basis of gene-specific nonhomologous DNA sequences. The standard curve method was used for quantification of the relative amounts of gene expression products. This method provides unitless normalized expression values that can be used for direct comparison of the relative amounts of target DNA and mRNA in different samples. We considered gastric cancer samples with RhoA mRNA expression decreased by more than $50 \%$. All samples were tested in duplicate, and average values were used for quantification.

\section{RhoA expression in gastric cancer tissues}

Expression of RhoA was examined in 35 frozen gastric cancers and corresponding noncancerous gastric tissues by Western blot analysis. These samples were ground to a very fine powder in liquid nitrogen with use of a mortar and pestle and suspended in an ice-cold Nonidet P-40 lysis buffer supplemented with a $1 \times$ protease inhibitor mix (Roche). Cell lysates were separated on $10 \%$ polyacrylamide gel and blotted onto a Hybond poly(vinylidene 
Table 1 The list of primers for real-time quantitative polymerase chain reaction

\begin{tabular}{ll}
\hline Gene & Primer \\
\hline GKN1 & F: CAAAGTCGATGACCTGAGCA \\
RhoA & R: CTTGCCTCTTGCATCTCCTC \\
& F: CAGAAAAGTGGACCCCAGAA \\
GAPDH & R: GCAGCTGCTCTCGTAGCCATTTC \\
miR-185_RT & F: AAATCAAGTGGGGCGATGCTG \\
miR-185 & R: GCAGAGATGATGACCCTTTTG \\
miR-34a_RT & GTCGTATCCAGTGCAGGGTCCGAGGTATTCGCACTGGATACGACTCAGGAA \\
miR-34a & F: CAATGGAGAGAAAGGCAGTTCC \\
U6 & R: AATCCATGAGAGATCCCTACCG \\
& GTCGTATCCAGTGCAGGGTCCGAGGTATTCGCACTGGATACGACAACAAC \\
RhoA_mutation 1 & F: GTGCAGGGTCCGAGGT \\
& R: CATGGCAGTGTCTTAGCTGGTT \\
RhoA_mutation 2 & F: ATTGGAACGATACAGAGAAGATT \\
& R: GGAACGCTTCACGAATTT \\
& F: CAGGTAATATCTGTGTTTTGT \\
& R: GCTTTCCATCCACCTCGATA \\
& F: GGGGGATTAACCTTGCACTC \\
& R: ACTATCAGGGCTGTCGATGG
\end{tabular}

$F$ forward, GAPDH glyceraldehyde 3-phosphate dehydrogenase, GKN1 gastrokine 1, $R$ reverse, $R T$ reverse transcribed

difluoride) transfer membrane (Amersham), which was subsequently probed with anti-RhoA monoclonal antibody (Abcam), and then incubated with anti-mouse IgG conjugated with horseradish peroxidase. The protein bands were detected with enhanced chemiluminescence Western blotting detection reagents (Amersham).

\section{Cell culture and transfection of $G K N 1$, anti-miR- 34a, and anti-miR-185}

AGS and MKN1 gastric cancer cells lines were cultured at $37{ }^{\circ} \mathrm{C}$ in $5 \% \mathrm{CO}_{2}$ in RPMI-1640 medium with $10 \%$ heatinactivated fetal bovine serum. The complete $G K N 1$ cDNA was cloned into the expression vector pcDNA3.1 (Invitrogen, Carlsbad, CA, USA). AGS and MKN1 Cells were transfected in $60 \mathrm{~mm}$-diameter dishes with GKN1 expression plasmids ( $5 \mu \mathrm{g}$ total DNA) and anti-miR-34a and antimiR-185 (100 nmol) by means of Lipofectamine Plus transfection reagent (Invitrogen) according to the manufacturer's recommendations.

\section{Sodium dodecyl sulfate-polyacrylamide gel electrophoresis and Western blot analysis}

To identify the expression of RhoA following GKN1 transfection, we examined the expression level of RhoA. The following antibodies were used; anti-GKN1 (SigmaAldrich, St Louis, MO, USA), anti-RhoA, anti-c-Myc, anti-
E-cadherin, anti-Snail, anti-Slug, anti-vimentin (Cell Signaling Technology, Danvers, MA, USA), anti-glyceraldehyde 3-phosphate dehydrogenase (Abcam, Cambridge, UK), anti-mouse $\operatorname{IgG}$, and anti-rabbit IgG (Sigma). An ECL Plus Western blotting detection system (Amersham) was used to detect the bound antibodies. The intensity of Western blot bands was quantified with an LAS 3000 system (Fujifilm, Japan).

\section{Measurement of cell migration and invasion assay}

To examine the effects of GKN1 and RhoA on cell migration and invasion, MKN1 gastric cancer cells were transfected with wild-type $G K N 1$ or small interfering RNA against RhoA (siRhoA) and treated with GKN1 recombinant protein (ANRT, Daejeon, Korea). Migration was assessed by an in vitro scratch wound-healing assay. A cell-free area was manually created by the scratching of the confluent cell monolayers with a $200-\mu l$ pipette tip.

Cell motility was assayed in 48-well micro chemotaxis chambers with gelatin-coated $8-\mu \mathrm{m}$ polyvinylpyrrolidinefree polycarbonate filters (Neuroprobe, Cabin John, MD, USA). A suspension containing $6 \times 10^{4}$ cells was added to each upper chamber, and the cell number was determined by calculation of the number of cells in the lower chamber in a $\times 20$ field from three independent experiments.

Invasion was assayed in Biocoat Matrigel invasion chambers (BD Bioscience, San Jose, CA, USA) with an 
$8-\mu \mathrm{m}$ pore polycarbonate filter coated with $0.5-\mathrm{mm}$ Matrigel according to the manufacturer's protocol. The membranes were fixed, stained with Diff-Quick (Sysmex, Kobe, Japan), and the number of cells was counted by direct visualization of nuclei in two $\times 20$ fields.

\section{Measurement of miR-34a and miR-185 expression}

Real-time reverse transcription PCR was performed with SYBR Green qPCR master mix (Stratagene, La Jolla, CA, USA) according to the manufacturer's instructions. To investigate whether GKN1 directly regulates miR-34a and miR-185 expression, expression of miR-34a and miR-185 was also quantified by qPCR and normalized to the level of human U6 small nuclear RNA. Data are reported as relative quantities according to an internal calibrator using the $2^{-\triangle \triangle \mathrm{Ct}}$ method [11]. The primer sequences are shown in Table 1.

\section{Statistical analysis}

Student's $t$ test was used to analyze the effect of GKN1 and RhoA on cell migration and invasion. All experiments were performed in duplicate to verify the reproducibility of the findings. Data are expressed as the mean \pm the standard deviation from at least two independent experiments. GKN1, RhoA, miR-34a, and miR-185 mRNA expression was examined with Wilcoxon rank sum tests. In addition, association between GKN1, RhoA, miR-34a, and miR-185 expression was examined with chi-square and Spearman correlation tests. A $P$ value less than 0.05 was considered the limit of statistical significance.

\section{Results}

\section{RhoA expression in gastric cancer tissues}

To investigate whether RhoA is involved in the progression of gastric cancer, we analyzed RhoA protein and mRNA expression in gastric cancer tissues and the corresponding noncancerous gastric mucosae. Among the 35 gastric cancer tissues tested, 15 (42.9\%), and 17 (48.6\%) showed increased RhoA protein and mRNA expression respectively (Fig. 1a, b). When the gastric cancers were stratified by differentiation, RhoA overexpression was observed in $8(34.8 \%)$ of 23 intestinal-type and $7(58.3 \%)$ of 12 diffuse-type gastric cancers, respectively. When we compared RhoA mRNA expression with T category and TNM stage of gastric cancer, RhoA expression was increased in gastric cancers with a higher $\mathrm{T}$ category and TNM stage (Fig. 1c).
Mutations of the RhoA gene in gastric cancer

We examined the gastric cancer tissues for the presence of any mutations that could be associated with the activation of RhoA using sequencing analysis. Unexpectedly, no RhoA mutations were detected in any of the 35 gastric cancer tissues. The experiments, including PCR and sequencing analysis, were repeated twice, to ensure the reliability of the results obtained (data not shown).

\section{GKN1 and RhoA expression in gastric cancer}

When we repeated the correlation with GKNl and RhoA gene expression levels in gastric cancer tissues, GKN1 mRNA expression was inversely correlated with $R h o A$ mRNA expression $(P<0.0001 ;$ Fig. $2 a)$. In addition, GKN1 expression was significantly decreased in 27 $(77.1 \%)$ of the 35 gastric cancers with a higher T category $(P=0.0001)$ but not in those with a higher TNM stage $(P=0.1790)$ (Fig. 2b). To analyze the effects of GKN1 on RhoA expression, we analyzed RhoA expression in AGS and MKN1 cells transfected with GKN1. We found that ectopic GKN1 expression markedly reduced RhoA mRNA and protein expression in both AGS and MKN1 cells (Fig. 2c, d), indicating that GKN1 downregulates RhoA expression in gastric cancer cells.

\section{The effect of GKN1-induced miR-185 on RhoA expression}

Since we have previously reported that GKN1 induced miR-185 expression in gastric cancer cells [11], and miR185 is a negative regulator of RhoA by directly binding to the $3^{\prime}$ untranslated region (UTR) of RhoA [20], we investigated whether GKN1 inhibits RhoA expression in a miR185-dependent manner. A significant increase in the expression of miR-185 was confirmed in AGS and MKN1 cells transfected with GKN1 (Fig. 3a). Additionally, the expression of miR-185 was dramatically decreased in 22 $(62.9 \%)$ of the 35 gastric cancer tissues (Fig. 3b). There was a positive correlation between GKN1 and miR-185 expression, and an inverse correlation between miR-185 and RhoA expression in gastric cancers (Fig. 3c, d). Specifically, we found that miR-185 inhibited RhoA expression by binding to a plausible recognition site in the $3^{\prime}$ UTR region of RhoA (Fig. 3e), and silencing of miR-185 with anti-miR-185 resulted in the recovery of the expression of RhoA in GKN1-transfected cells (Fig. 3f). Taken together, these results indicate that GKN1 inhibits RhoA expression through the upregulation of miR-185 expression. 
Fig. 1 Expression of RhoA in gastric cancer tissues. a, b Expression of RhoA protein and messenger RNA (mRNA) was significantly increased in gastric cancer tissues. The Western blot is a representative result of two individual experiments. $\mathbf{c}$ Increased expression of RhoA mRNA transcript was detected in the gastric cancers with a higher $\mathrm{T}$ category and TNM stage $(P<0.0001$ and $P=0.0009$ respectively). The relative expression levels of RhoA mRNA in noncancerous gastric mucosal tissues (normal) and gastric cancer (tumor) are illustrated by a scatter plot. The data shown are the mean value of two individual experiments. The median expression level of each group is indicated by a horizontal line. GAPDH glyceraldehyde 3-phosphate dehydrogenase, $N$ normal, $T$ tumor

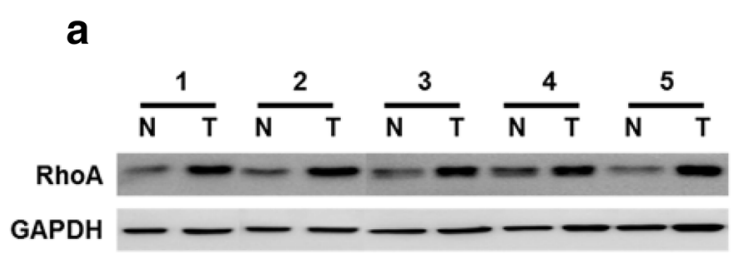

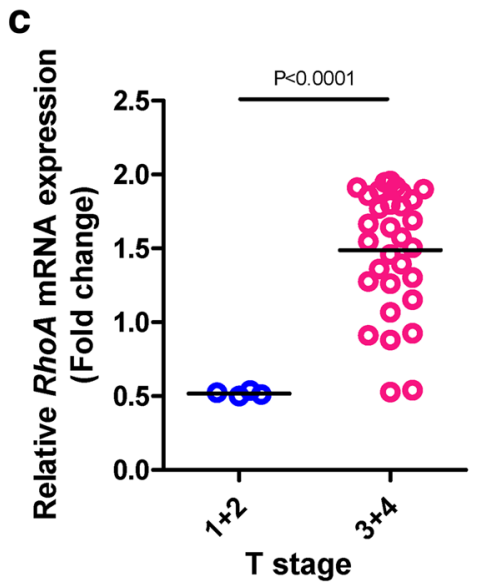
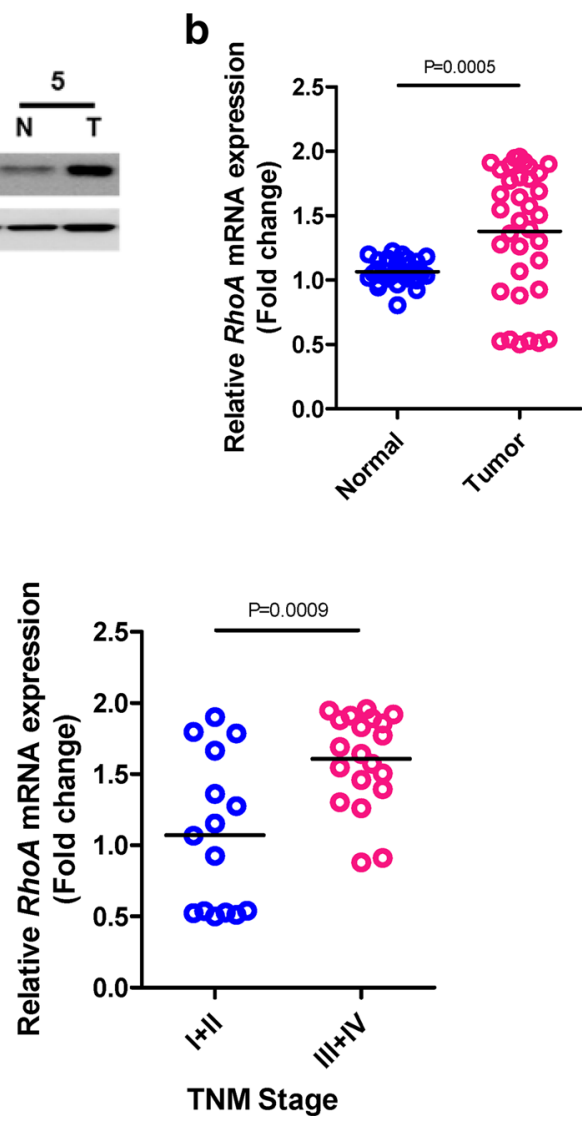

\section{The effect of GKN1-induced miR-34a on RhoA expression}

c-Myc is an important transcription factor for RhoA transcription [21] and represses miR-34a expression [22]. Therefore, we further investigated whether GKN1 regulates RhoA expression by upregulating miR-34a expression. Expression of miR-34a was decreased in $7(20 \%)$ of the 35 gastric cancer tissues (Fig. 4a) and was positively correlated with GKN1 and miR-185 expression and inversely correlated with RhoA expression (Fig. 4b). In AGS and MKN1 cells, ectopic GKN1 expression induced miR34a expression (Fig. 4c). GKN1 suppressed c-Myc and RhoA expression, whereas miR-34a with anti-miR-34a silencing induced reexpression of c-Myc and RhoA in GKN1-transfected cells (Fig. 4d). As shown in Fig. 4e, miR-34a inhibited c-Myc expression by binding to a plausible recognition site in the $3^{\prime}$ UTR region of c-Myc. Furthermore, miR-34a silencing led to decreased expression of miR-185 (Fig. 4f), indicating that GKN1 may inhibit c-Myc and RhoA expression through the upregulation of miR-34a expression.

\section{The effects of GKN1 and RhoA on gastric cancer cell migration and invasion}

To determine whether GKN1 and RhoA contribute to gastric cancer cell migration and invasion, we performed in vitro wound healing, Transwell chemotaxis, and Matrigel invasion assays. Ectopic GKN1 expression, silencing of RhoA, and recombinant GKN1 treatment of MKN1 cells markedly decreased cell migration activity into the wound area, compared with that of mock transfected cells (Fig. 5a). In addition, ectopic GKN1 expression and silencing of RhoA significantly suppressed cell migration in the Transwell microchemotaxis assays at 2 days after culture (Fig. 5b). In a Matrigel assay, invasiveness of gastric cancer cells was also dramatically inhibited in GKN1- and siRhoA-transfected cells at 2 days after culture (Fig. 5c). Since EMT is a critical process in cell migration and invasion [23, 24], we further analyzed the role of GKN1 and RhoA in the expression of EMT-related proteins. In MKN1 gastric cancer cells, ectopic GKN1 expression, or silencing of RhoA, increased E-cadherin expression, and decreased the expression of RhoA, Snail, Slug, and vimentin (Fig. 5d). Notably, treatment with 
Fig. 2 Gastrokine $1(G K N 1)$ negatively regulates RhoA expression. a An inverse association between $G K N 1$ messenger RNA ( $m R N A)$ expression and RhoA mRNA expression was observed in gastric cancer tissues. The data shown are mean values of at least two individual experiments. b Decreased expression of GKN1 mRNA transcript was closely associated with a higher $\mathrm{T}$ category $(P=0.0001)$ but not with a higher TNM stage

$(P=0.1790)$. c Ectopic GKN1 expression in AGS and MKN1 cells significantly

downregulated RhoA mRNA transcript expression. d Western blot analysis following GKN1 transfection in AGS and MKN1 cells showed a downregulation of RhoA expression. The blot is a representative result of two individual experiments. $G A P D H$ glyceraldehyde 3-phosphate dehydrogenase a

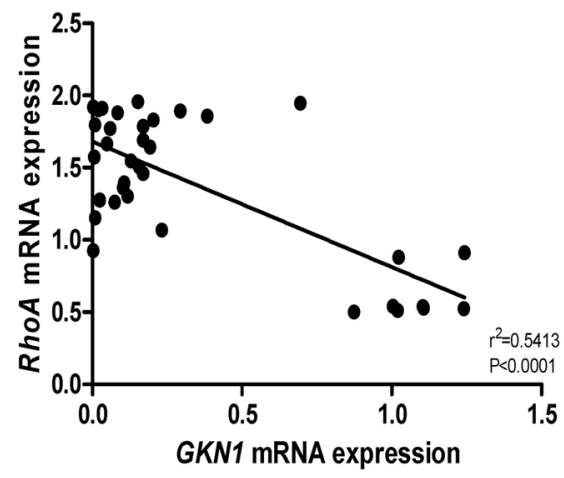

b

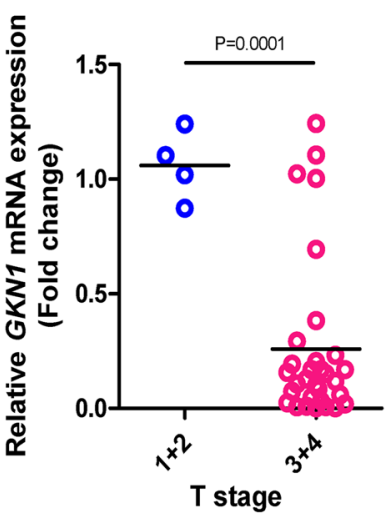

C

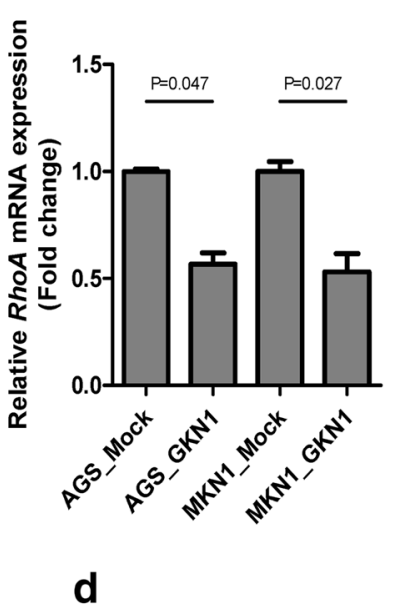

recombinant GKN1 increased E-cadherin expression, and decreased the expression of the EMT-related proteins Snail, Slug, and vimentin in a dose-dependent manner (Fig. 5e). Furthermore, silencing of miR-185 and miR-34a in MKN1 cells transfected with GKN1 increased cell migration, invasion, and expression of the EMT-related proteins Snail, Slug, and vimentin (Fig. 6), suggesting that GKN1-induced miR185 and miR-34a expression may inhibit gastric cancer cell migration and invasion by inactivating RhoA.

\section{Discussion}

RhoA was found to be frequently overexpressed in 15 $(42.9 \%)$ of the gastric cancer tissues tested compared with the corresponding noncancerous gastric mucosae, and its overexpression was closely associated with more advanced pathological $\mathrm{T}$ category and TNM stage (Fig. 1). These results are consistent with those of previous studies [25, 26] and suggest that RhoA is involved in the progression of gastric cancers, acting as an oncogene. When the gastric cancers were stratified by differentiation, we found no significant difference in RhoA overexpression between intestinal-type and diffuse-type gastric cancers, indicating that RhoA contributes to the tumorigenesis of both types of gastric cancers.

Recently, RhoA mutations have been detected in gastric cancers, especially of diffuse type [27-29]. From these observations, it is possible that mutant RhoA might cause defective RhoA signaling, which would promote cancer cell migration and invasion, especially in diffuse-type gastric cancer. In contrast, no somatic mutation of RhoA was detected in this study, suggesting that somatic mutations may not be a cause of RhoA activation in these gastric cancers (data not shown). As the gastric specific tumor suppressor GKN1 was frequently downregulated in gastric cancers [19], we analyzed GKN1 inactivation as a possible cause of RhoA overexpression. In our analysis of $R h o A$ and GKN1 expression in gastric cancers, GKN1 mRNA expression was inversely correlated with $R h o A$ expression (Fig. 2a), and markedly decreased in gastric cancers with a higher $\mathrm{T}$ category and TNM stage (Fig. 2b). In addition, ectopic expression of GKN1 in AGS and MKN1 cells dramatically downregulated RhoA expression (Fig. 2c, d). These results further support the idea that deregulation of RhoA signaling may be caused by genomic abnormalities 
Fig. 3 Gastrokine 1 (GKNl) negatively regulates RhoA expression by upregulating miR-185. a Ectopic GKN1 expression in AGS and MKN1 cells markedly induced miR185 expression. b Expression of miR-185 was significantly decreased in gastric cancer tissues $(P<0.0001)$. The relative expression levels of miR-185 in noncancerous gastric mucosal tissues (normal) and gastric cancer (tumor) are illustrated by a scatter plot. The median expression level of each group is indicated by a horizontal line. c Positive correlation between GKN1 and miR-185 expression was found in the 35 gastric cancer tissues. d An inverse association of GKN1 expression with miR-185 expression was observed in gastric cancer tissues. e Prediction schema for the binding sites between miR-185 and the $3^{\prime}$ untranslated region of RhoA. f GKN1-transfected AGS and MKN1 cells showed complete downregulation of RhoA expression, but cotransfection of GKN1 and anti-miR-185 resulted in recovery of RhoA expression. The blot is a representative result of two individual experiments. GAPDH glyceraldehyde 3-phosphate dehydrogenase a

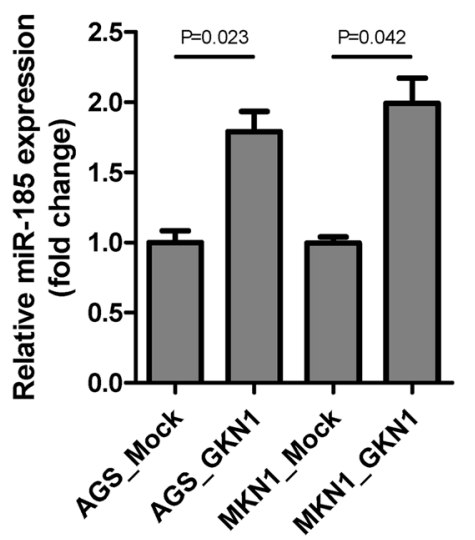

C

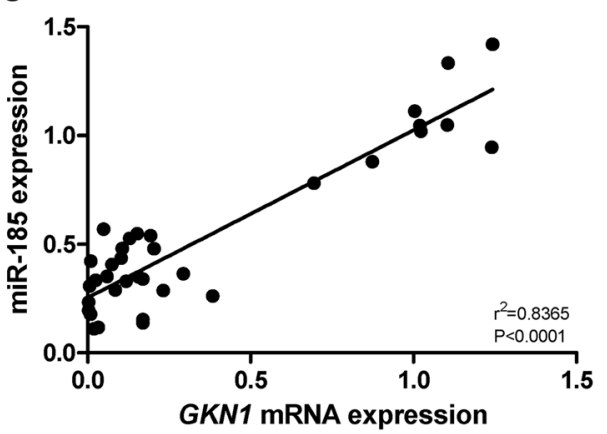

d
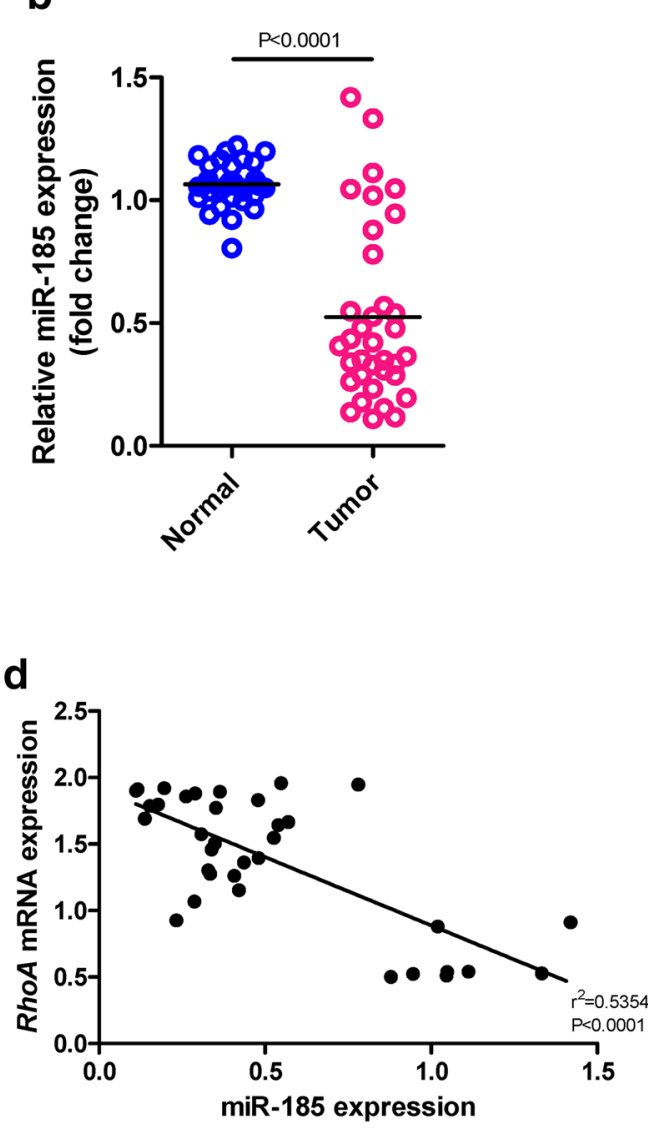

e

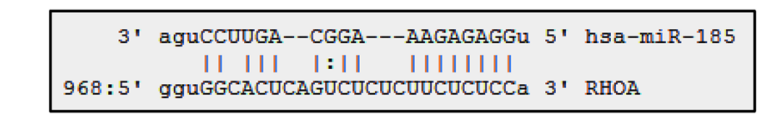

f

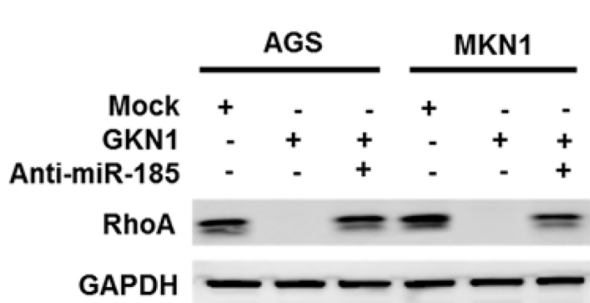

other than RhoA mutations [30], and suggest that GKN1 possesses a tumor suppressive capability, through the inactivation of RhoA.

Next, to identify the potential molecular mechanisms involved in the regulation of RhoA expression by GKN1, we analyzed miR-185, which is known to directly target RhoA [20]. We had previously found that GKN1 induces miR-185 expression in gastric epithelial cells and that miR185 plays an inhibitory role in cell proliferation [11]. In the present study, we have shown that GKN1 induces miR-185 expression in AGS and MKN1 gastric cancer cells (Fig. 3a). In addition, miR-185 expression was significantly decreased in gastric cancers (Fig. 3b), and its expression was positively associated with GKN1 expression and inversely correlated with RhoA expression (Fig. 3c, d). Furthermore, we found that GKN1-induced miR-185 inhibits RhoA expression by binding to a plausible recognition site in the $3^{\prime}$ UTR region of RhoA (Fig. 3e). In AGS and MKN1 gastric cancer cells, ectopic GKN1 expression completely inhibited RhoA expression, 
Fig. 4 Gastrokine 1 (GKN1) negatively regulates expression of RhoA by upregulating miR34a. a Decreased expression of miR-34a was observed in gastric cancer tissues. The relative expression levels of miR-34a in noncancerous tissues (normal) and gastric cancer (tumor) are illustrated by a scatter plot. The median expression level of each group is indicated by a horizontal line. b Positive correlation of miR34a expression with GKN1 and miR-34a expression and an inverse association with $R h o A$ expression were found in the 35 gastric cancer tissues. c Ectopic GKN1 expression in AGS and MKN1 cells induced miR-34a expression. d GKN1-transfected AGS and MKN1 cells showed downregulation of c-Myc and RhoA expression, but cotransfection of GKN1 and anti-miR-34a restored the expression. The blot is a representative result of two individual experiments. e Prediction schema of the binding sites between miR-34a and the $3^{\prime}$ untranslated region of c-Myc. f GKN1-transfected AGS and MKN1 cells dramatically increased miR-185 expression, whereas cotransfection of GKN1 and anti-miR-34a downregulated the GKN1-induced miR-185 expression. We repeated the experiments twice, and demonstrated consistency a

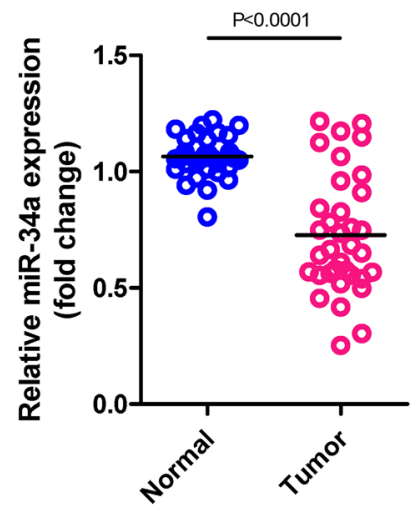

C

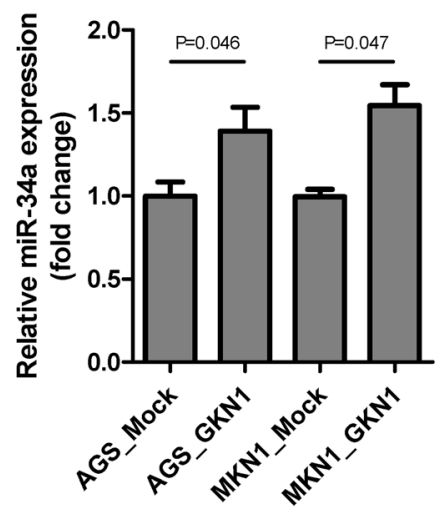

d

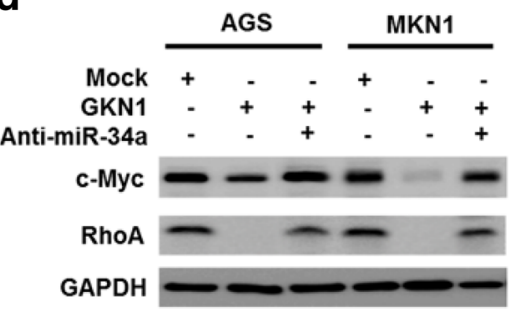

e

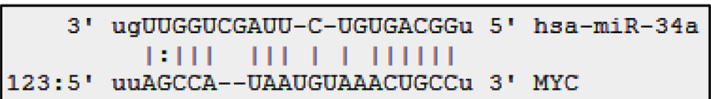

b
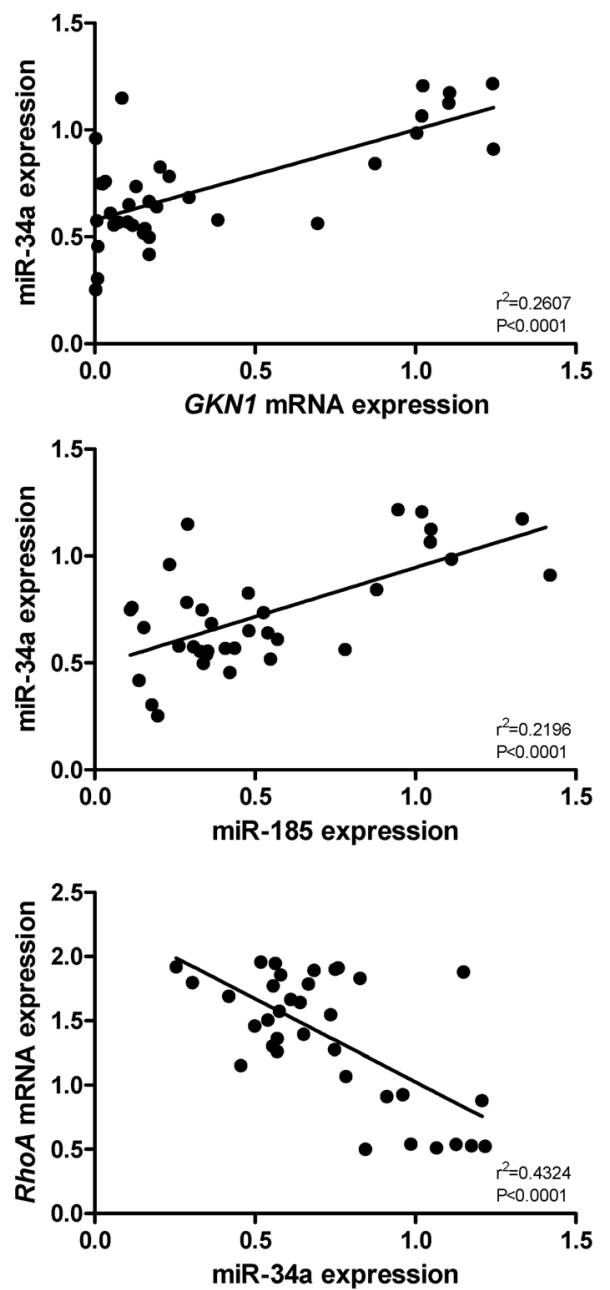

f

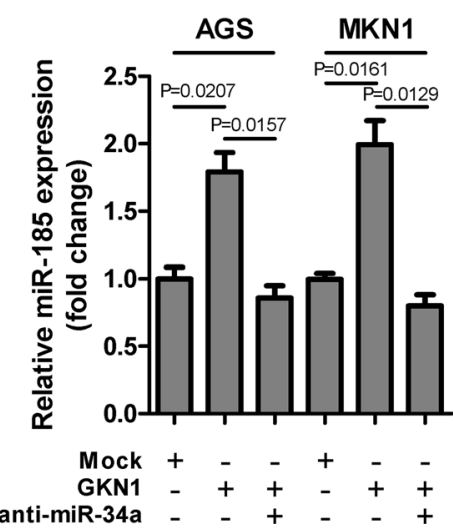

whereas miR-185 silencing, with anti-miR-185, restored the GKN1-induced RhoA expression (Fig. 3f), suggesting that GKN1-induced miR-185 may inhibit gastric carcinogenesis by downregulating RhoA expression.
Interestingly, miR-34a, a pivotal member of the p53 network, was found to be downregulated in several types of tumor, and was reported to act as a tumor suppressor [31]. In addition, miR-34a inhibited tumor invasion and 
Fig. 5 Gastrokine 1 (GKNl) inhibits cell migration and invasion by inactivating RhoA. a In the wound healing assay, a significant inhibition of cell migration was observed in MKN1 cells $24 \mathrm{~h}$ after treatment with GKN1 or RhoA small interfering RNA (siRhoA) or recombinant GKN1 $(r G K N 1)$. b, c In chemotaxis and Matrigel assays, GKN1- or siRhoA-transfected MKN1 cells markedly inhibited cell migration and invasion. d Ectopic GKN1 expression and RhoA silencing in MKN1 cells upregulated E-cadherin expression and downregulated the expression of epithelial-tomesenchymal transition related proteins, including Snail, Slug, and vimentin. e Western blot analysis following recombinant GKN1 treatment in MKN1 cells showed an increase in E-cadherin expression but a decrease in the expression of RhoA, Snail, Slug, and vimentin in a dose-dependent manner. GAPDH glyceraldehyde

3-phosphate dehydrogenase a
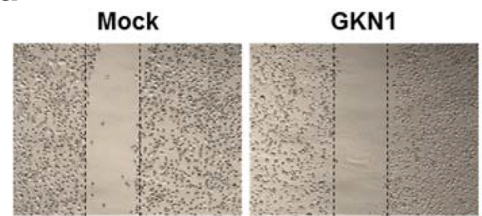

siRhoA

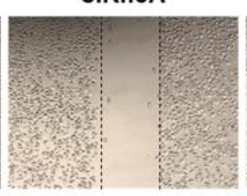

rGKN1-treated
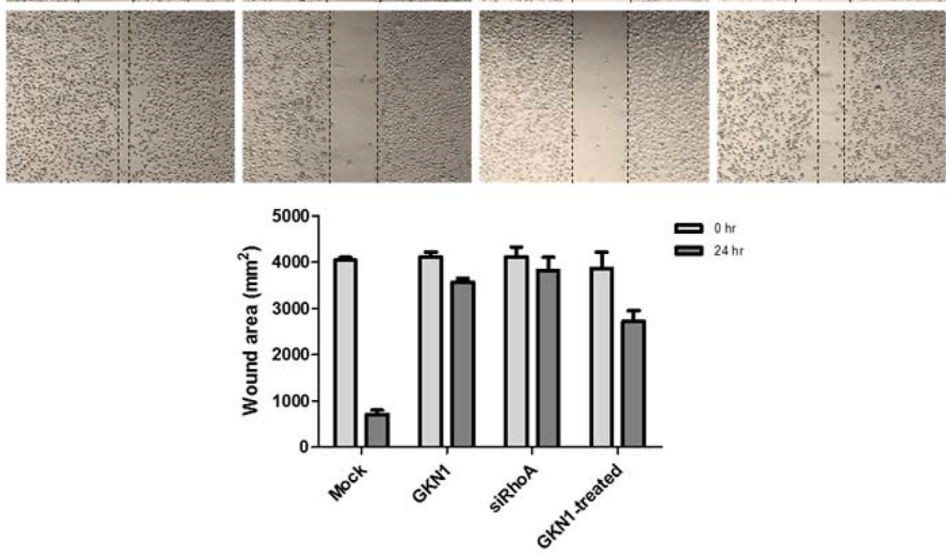

b
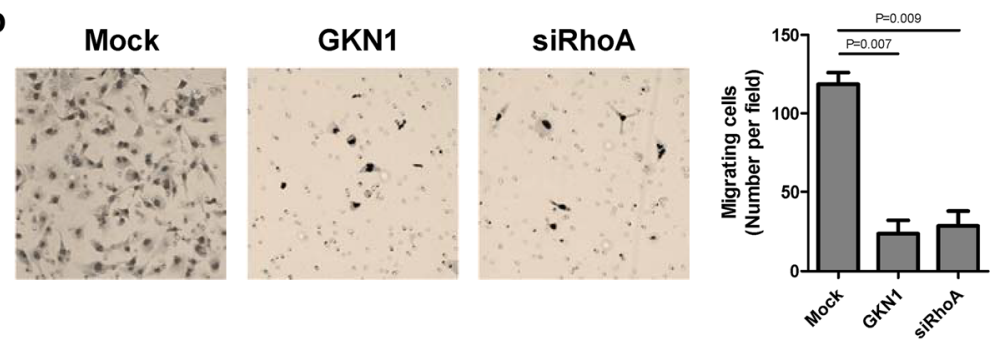

C
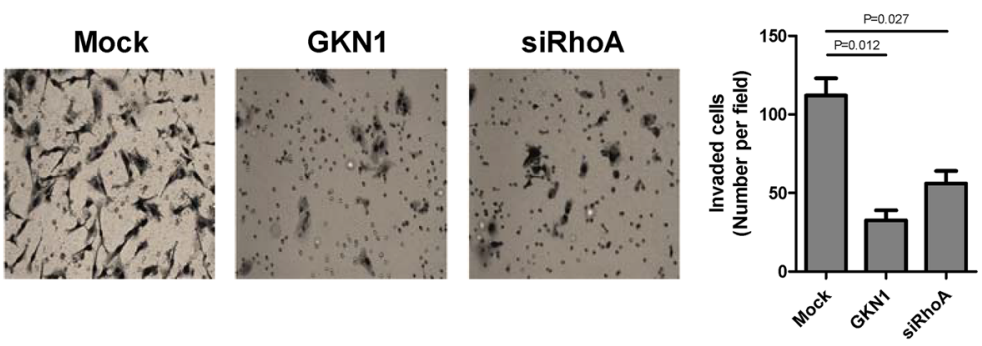

d

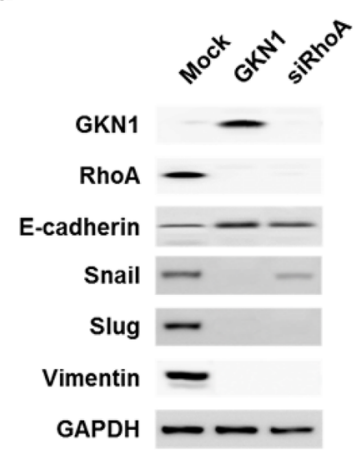

e

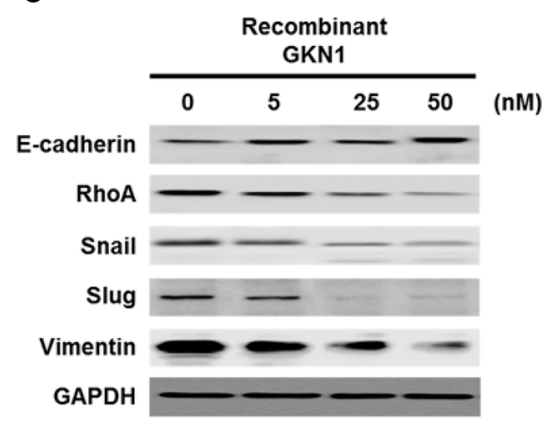

metastasis in gastric cancer by targeting Tgif2 [32]. As GKN1 downregulates c-Myc expression, and miR-34a directly targets c-Myc [33], we investigated whether GKN1 induces miR-34a expression, and how miR-34a regulates the expression of RhoA. As expected, miR-34a expression was significantly decreased in gastric cancers (Fig. 4a), and 
a

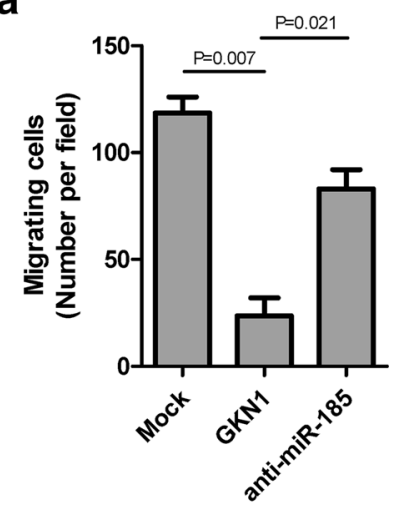

b

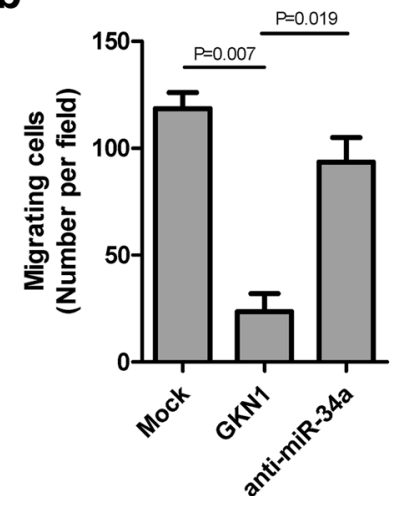

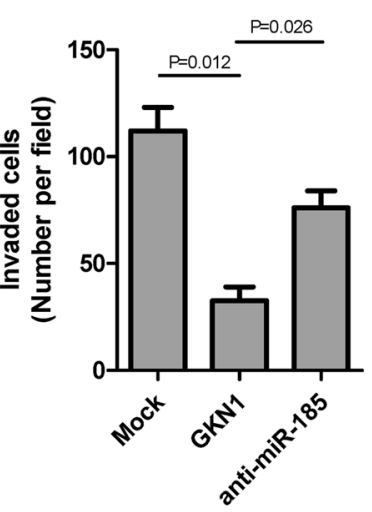

C

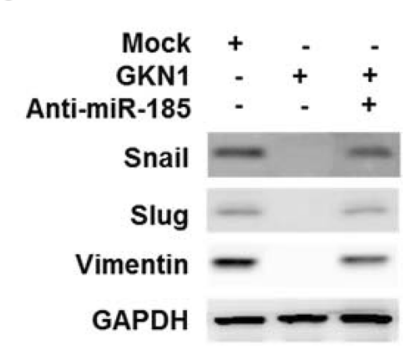

d

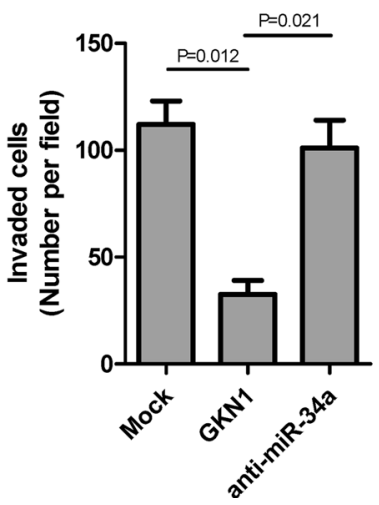

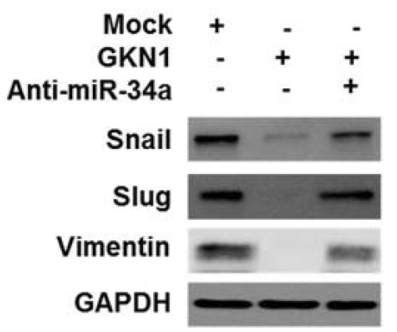

Fig. 6 Gastrokine $1(G K N 1)$ inhibits cell migration and invasion in a miR-185- and miR-34a-dependent manner. a GKN1-transfected MKN1 cells significantly inhibited cell migration and invasion, but miR-185 silencing with anti-miR-185 resulted in recovery of cell migration and invasion. b GKN1-transfected MKN1 cells significantly inhibited cell migration and invasion, but miR-34a silencing with anti-miR-34a resulted in recovery of cell migration and invasion. c,

its expression was positively associated with $G K N 1$ and miR-185 expression and inversely correlated with RhoA expression in gastric cancers (Fig. 4b). As shown in Fig. 4c and $\mathrm{d}$, GKN1 upregulated the expression of miR-34a and decreased the expression of c-Myc and RhoA. We also found a plausible binding site of miR-34a in the $3^{\prime}$ UTR region of c-Myc (Fig. 4e). When we c-transfected AGS and MKN1 cells with GKN1 and anti-miR-34a, GKN1-induced expression of miR-185 was markedly inhibited (Fig. 4f). c-Myc inhibits miR-185 expression by binding to a promoter of miR-185 [11], and as such it is likely that c-Myc expression, induced by miR-34a silencing, leads to the inhibition of miR-185 expression.

Since RhoA primarily mediates signaling pathways related to cell growth, transformation, and migration [34], we investigated whether GKN1 inhibits RhoA-induced EMT, cell migration, and invasion. MKN1 gastric cancer cells were also subjected to small interfering RNA mediated silencing of RhoA to explore the role of RhoA in d GKN1-transfected MKN1 cells significantly downregulated the expression of Snail, Slug, and vimentin; however, silencing of miR185 and miR-34a in GKN1-transfected MKN1 cells restored the expression of these epithelial-to-mesenchymal transition related proteins. The blot is a representative result of two individual experiments. GAPDH glyceraldehyde 3-phosphate dehydrogenase. $G A P D H$ glyceraldehyde 3-phosphate dehydrogenase

gastric cancer in vitro. A near complete inhibition of cell migration was observed in GKN1- and siRhoA-transfected cells and cells treated with recombinant GKN1 (Fig. 5a). In the chemotaxis and Matrigel assays, ectopic GKN1 expression and silencing of RhoA with siRhoA significantly suppressed cell migration and invasion (Fig. 5b, c). In addition, GKN1 expression and RhoA silencing induced E-cadherin reexpression, and elicited suppression of RhoA, Snail, Slug, and vimentin expression (Fig. 5d). Moreover, treatment with recombinant GKN1 modulated expression of these EMT-related proteins in a dose-dependent manner (Fig. 5e). These results are consistent with the data showing that RhoA silencing inhibits proliferation and migration of MGC-803 gastric cells [35], and suggest that GKN1 may suppress gastric carcinogenesis by downregulating RhoA expression, and as such may be an effective therapeutic target for gastric cancer.

Determining factors underlying gastric cancer cell invasion is important for a general understanding of the 
progression and treatment of gastric cancer. Recently, it has been reported that RhoA activation is required for Wnt5a/PI3K/Akt/glycogen synthase kinase $3 \beta$ activity and that Wnt5a/PI3K/Akt/glycogen synthase kinase 33/RhoA signaling may contribute to cell migration [36]. Our previous study demonstrated that GKN1 inhibits gastric cancer migration and invasion through suppression of reactive oxygen species and the PI3K/Akt pathway [12]. Here, the fact that GKN1 inhibits RhoA expression implies that GKN1-induced miR-185 and miR-34a expression can modulate gastric cancer cell migration and invasion. Notably, silencing of miR-185 and miR-34a restored the cell migration and invasion that were significantly inhibited by GKN1 (Fig. 6a, b). Additionally, GKN1 completely suppressed the expression of EMT-related proteins, whereas silencing of miR-185 and miR-34a induced their expression (Fig. 6c, d). Hence, these findings suggest that GKN1 inhibits gastric cancer cell migration and invasion via both inactivating the PI3K/Akt signaling pathway and targeting RhoA by upregulating miR-185 and miR-34a. Further studies are needed to identify the effects of miR34a and miR-185 on the PI3K/Akt signaling pathway.

In summary, we have shown that the expression of the gastric tumor suppressor GKN1 and that of miR-185 and miR-34a were downregulated and inversely correlated with RhoA expression in the 35 gastric cancer tissues tested. In gastric cancer cell lines, GKN1 induced miR-185 and miR34a expression, and demonstrated a substantial inhibition of gastric cancer cell migration and invasion by directly binding to RhoA. Therefore, we conclude that GKN1 could inhibit the progression of gastric cancer via its negative regulation of RhoA expression, and may be a novel therapeutic target for gastric cancer.

Acknowledgments This work was supported by the Basic Science Research Program through the National Research Foundation of Korea funded by the Ministry of Education, Science and Technology (2015R1A2A2A05001023). We thank Seong Yeob Ryu, Department of Gastroenterologic Surgery, Chonnam National University Hwasun Hospital, Korea, for providing the gastric cancer samples with clinical information.

\section{Compliance with ethical standards}

Conflict of interest The authors disclose no potential conflicts of interest.

Informed consent statement All study participants, or their legal guardian, provided informed written consent before study enrollment.

\section{References}

1. Jemal A, Bray F, Center MM, Ferlay J, Ward E, Forman D. Global cancer statistics. CA Cancer J Clin. 2011;61:69-90.
2. Ferlay J, Soerjomataram I, Ervik M, Dikshit R, Eser S, Mathers C, et al. GLOBOCAN 2012 v1.0, Cancer incidence and mortality worldwide: IARC CancerBase no. 11. International Agency for Research on Cancer, Lyon. 2013. Available from http://globocan. iarc.fr. Accessed 17 Apr 2015.

3. Camargo MC, Kim WH, Chiaravalli AM, Kim KM, Corvalan $\mathrm{AH}$, Matsuo K, et al. Improved survival of gastric cancer with tumour Epstein-Barr virus positivity: an international pooled analysis. Gut. 2014;63:236-43.

4. Deng JY, Liang H. Clinical significance of lymph node metastasis in gastric cancer. World J Gastroenterol. 2014;20:3967-75.

5. Kalluri R, Weinberg RA. The basics of epithelial-mesenchymal transition. J Clin Invest. 2009;119:1420-8.

6. Chen J, Wang T, Zhou YC, Gao F, Zhang ZH, Xu H, et al. Aquaporin 3 promotes epithelial-mesenchymal transition in gastric cancer. J Exp Clin Cancer Res. 2014;33:38.

7. Xiang Z, Jiang DP, Xia GG, Wei ZW, Chen W, He Y, et al. CXCL1 expression is correlated with Snail expression and affects the prognosis of patients with gastric cancer. Oncol Lett. 2015;10:2458-64.

8. Li H, Wang Z, Zhang W, Qian K, Xu W, Zhang S. Fbxw7 regulates tumor apoptosis, growth arrest and the epithelial-to-mesenchymal transition in part through the RhoA signaling pathway in gastric cancer. Cancer Lett. 2016;370:39-55.

9. Oien KA, McGregor F, Butler S, Ferrier RK, Downie I, Bryce S, et al. Gastrokine 1 is abundantly and specifically expressed in superficial gastric epithelium, down-regulated in gastric carcinoma, and shows high evolutionary conservation. J Pathol. 2004;203:789-97.

10. Walsh-Reitz MM, Huang EF, Musch MW, Chang EB, Martin TE, Kartha S, et al. AMP-18 protects barrier function of colonic epithelial cells: role of tight junction proteins. Am J Physiol Gastrointest Liver Physiol. 2005;289:G163-71.

11. Yoon JH, Choi YJ, Choi WS, Ashktorab H, Smoot DT, Nam SW, et al. GKN1-miR-185-DNMT1 axis suppresses gastric carcinogenesis through regulation of epigenetic alteration and cell cycle. Clin Cancer Res. 2013;19:4599-610.

12. Yoon JH, Kang YH, Choi YJ, Park IS, Nam SW, Lee JY, et al. Gastrokine 1 functions as a tumor suppressor by inhibition of epithelial-mesenchymal transition in gastric cancers. J Cancer Res Clin Oncol. 2011;137:1697-704.

13. Xing R, Cui JT, Xia N, Lu YY. GKN1 inhibits cell invasion in gastric cancer by inactivating the NF-kappaB pathway. Discov Med. 2015;19:65-71.

14. Ridley AJ, Schwartz MA, Burridge K, Firtel RA, Ginsberg MH, Borisy $\mathrm{G}$, et al. Cell migration: integrating signals from front to back. Science. 2003;302:1704-9.

15. Thumkeo D, Watanabe S, Narumiya S. Physiological roles of Rho and Rho effectors in mammals. Eur $\mathrm{J}$ Cell Biol. 2013;92:303-15.

16. Takai Y, Sasaki T, Matozaki T. Small GTP-binding proteins. Physiol Rev. 2001;81:153-208.

17. Sahai E, Marshall CJ. RHO-GTPases and cancer. Nat Rev Cancer. 2002;2:133-42.

18. Karlsson R, Pedersen ED, Wang Z, Brakebusch C. Rho GTPase function in tumorigenesis. Biochim Biophys Acta. 2009;1796:91-8.

19. Yoon JH, Song JH, Zhang C, Jin M, Kang YH, Nam SW, et al. Inactivation of the gastrokine 1 gene in gastric adenomas and carcinomas. J Pathol. 2011;223:618-25.

20. Liu M, Lang N, Chen X, Tang Q, Liu S, Huang J, et al. miR-185 targets RhoA and $\mathrm{Cdc} 42$ expression and inhibits the proliferation potential of human colorectal cells. Cancer Lett. 2011;301:151-60. 
21. Chan CH, Lee SW, Li CF, Wang J, Yang WL, Wu CY, et al. Deciphering the transcriptional complex critical for RhoA gene expression and cancer metastasis. Nat Cell Biol. 2010;12:457-67.

22. Chang TC, Yu D, Lee YS, Wentzel EA, Arking DE, West KM, et al. Widespread microRNA repression by Myc contributes to tumorigenesis. Nat Genet. 2008;40:43-50.

23. Scheel C, Weinberg RA. Cancer stem cells and epithelial-mesenchymal transition: concepts and molecular links. Semin Cancer Biol. 2012;22:396-403.

24. Puisieux A, Brabletz T, Caramel J. Oncogenic roles of EMTinducing transcription factors. Nat Cell Biol. 2014;16:488-94.

25. Huang KH, Lan YT, Chen MH, Chao Y, Lo SS, Li AF, et al. The correlation between RhoA expression and clinicopathological characteristics in gastric cancer patients after curative surgery. World J Surg. 2015;39:2289-99.

26. Pan Y, Bi F, Liu N, Xue Y, Yao X, Zheng Y, et al. Expression of seven main Rho family members in gastric carcinoma. Biochem Biophys Res Commun. 2004;315:686-91.

27. Kakiuchi M, Nishizawa T, Ueda H, Gotoh K, Tanaka A, Hayashi A, et al. Recurrent gain-of-function mutations of RHOA in diffuse-type gastric carcinoma. Nat Genet. 2014;46:583-7.

28. Cancer Genome Atlas Research Network. Comprehensive molecular characterization of gastric adenocarcinoma. Nature. 2014;513:202-9.

29. Wang K, Yuen ST, Xu J, Lee SP, Yan HH, Shi ST, et al. Wholegenome sequencing and comprehensive molecular profiling identify new driver mutations in gastric cancer. Nat Genet. 2014;46:573-82.

30. Ushiku T, Ishikawa S, Kakiuchi M, Tanaka A, Katoh H, Aburatani $\mathrm{H}$, et al. RHOA mutation in diffuse-type gastric cancer: a comparative clinicopathology analysis of 87 cases. Gastric Cancer. 2016;19:403-11.

31. Li L, Yuan L, Luo J, Gao J, Guo J, Xie X. MiR-34a inhibits proliferation and migration of breast cancer through down-regulation of Bcl-2 and SIRT1. Clin Exp Med. 2013;13:109-17.

32. Hu Y, Pu Q, Cui B, Lin J. MicroRNA-34a inhibits tumor invasion and metastasis in gastric cancer by targeting Tgif2. Int J Clin Exp Pathol. 2015;8:8921-8.

33. Yamamura S, Saini S, Majid S, Hirata H, Ueno K, Deng G, et al. MicroRNA-34a modulates c-Myc transcriptional complexes to suppress malignancy in human prostate cancer cells. PLoS One. 2012;7:e29722.

34. Wennerberg K, Der CJ. Rho-family GTPases: it's not only Rac and Rho (and I like it). J Cell Sci. 2004;117:1301-12.

35. Duan JT, Wang XM, Zhang SQ, Zhao GJ. Effect of RhoA gene silencing on proliferation and migration of gastric MGC-803 cells. Int J Clin Exp Med. 2015;8:14410-5.

36. Liu J, Zhang Y, Xu R, Du J, Hu Z, Yang L, et al. PI3K/Aktdependent phosphorylation of GSK3 $\beta$ and activation of RhoA regulate Wnt5a-induced gastric cancer cell migration. Cell Signal. 2013;25:447-56. 